\title{
Terahertz Microchip for Illicit Drug Detection
}

\author{
Ja-Yu Lu, Li-Jin Chen, Tzeng-Fu Kao, Hsu-Hao Chang, Hung-Wen Chen, An-Shyi Liu, Yi-Chun Chen,
} Ruey-Beei Wu, Senior Member, IEEE, Wei-Sheng Liu, Jeng-Inn Chyi, and Chi-Kuang Sun, Senior Member, IEEE

\begin{abstract}
To accurately detect minute amounts of substances without disturbing the surroundings of target molecules has been a major goal in bioanalytical technology. Here by integrating an optoelectronic terahertz ( $\mathrm{THz})$ microsource into a glass-substrated microchip within the near-field distance, we demonstrate a compact, label-free, noninvasive, and sensitive microbiosensing system with low-power consumption. The demonstrated $\mathrm{THz}$ microchip allows us to locally specify various illicit drug powders with weights of nanograms, with a promising future for rapid identification of the static status or even the dynamics of various biomolecules.
\end{abstract}

Index Terms-Biochip, far-infrared, microchip, millimeterwave, photonic transmitter, submillimeter wave, terahertz (THz).

$\mathbf{S}$ ENSING devices designed to identify minute amounts of biochemical substances accurately and noninvasively are strongly desired for many applications, such as in lab-on-a-chip development. Current analytic methods, including fluorescencebased techniques, require modification of the surroundings of molecules, thus disturbing the nature of the target molecules that could lower the precision of detection [1], [2]. One alternative without disturbance of target molecules is the terahertz (THz)-based biosensing technique [2]-[6]. Based on molecular fingerprints, direct THz-probing is a sensitive, label-free, and noninvasive way for biomolecule detection. It is thus highly desirable to directly integrate a $\mathrm{THz}$ microsource with the biochip system within the near-field distance for highly sensitive and localized detection of biomaterials in channels. Although applying $\mathrm{THz}$ technologies to lab-on-a-chip is beneficial, the challenge that the glass-substrate of most biochips is much absorptive at the $\mathrm{THz}$ range must be overcome. A high sensitivity $\mathrm{THz}$ sensing technique was demonstrated by Kurz's group, which used an integrated planar microwave waveguide based on microstrip line resonantors to detect the DNA binding state (as a function of refractive index) [2], [4]. However, modification of the deposited DNA on the planar waveguides is required. Moreover, the demonstrated system cannot be used repeatably and is less suitable for planar integration with a wide variety of currently available biochip modules.

Manuscript received March 6, 2006; revised July 2, 2006. This work was supported by the National Science Council of Taiwan under Grant NSC94-2215-E002-007 and by the NTU Center for Genomic Medicine.

J.-Y. Lu, L.-J. Chen, T.-F. Kao, H.-H. Chang, H.-W. Chen, and C.-K. Sun are with the Department of Electrical Engineering and Graduate Institute of ElectroOptical Engineering, National Taiwan University, Taipei 10617, Taiwan, R.O.C. (e-mail: sun@cc.ee.ntu.edu.tw).

A.-S. Liu, Y.-C. Chen, and R.-B. Wu are with the Department of Electrical Engineering, National Taiwan University, Taipei 10617, Taiwan, R.O.C.

W.-S. Liu and J.-I. Chyi are with the Optical Sciences Center, National Central University, Chung-Li 32054, Taiwan, R.O.C.

Digital Object Identifier 10.1109/LPT.2006.883285

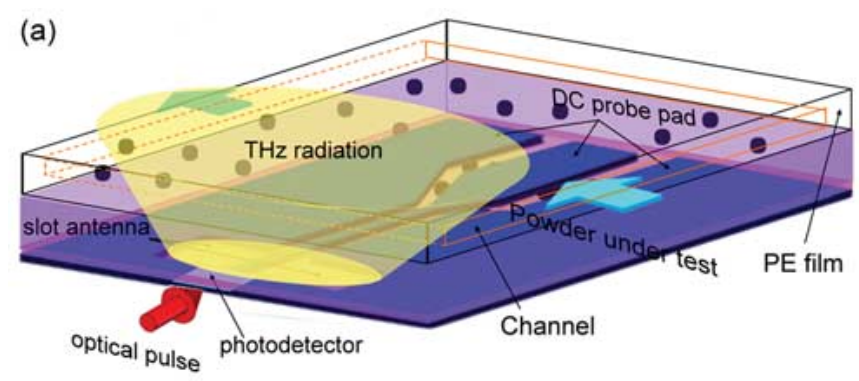

(b)

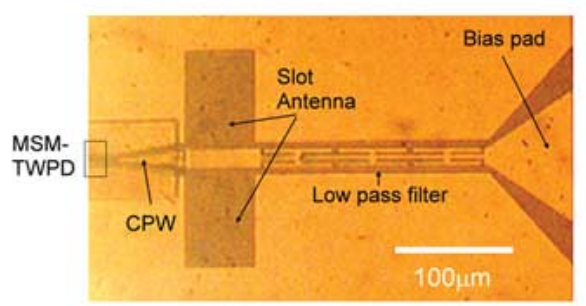

Fig. 1. (a) THz-integrated biosensing chip which is composed of a PE sample cell with microchannels therein, a $150-\mu \mathrm{m}$-thick glass substrate, and an integrated membrane-type $\mathrm{THz}$ photonic transmitter on the bottom of the glass substrate with a thickness of $5 \mu \mathrm{m}$. (b) Bottom view of the device showing the electronic circuit of the photonic transmitter [9].

In this letter, we demonstrate the feasibility to directly integrate an optoelectronic micro- $\mathrm{THz}$ source into a glasssubstrated microchip for direct, sensitive, and localized sensing of biomolecules, which are preserved in their native states. Through optical control [7], the near-field $\mathrm{THz}$ absorption spectra of the molecules inside the microchip can be directly acquired while different biomolecules can be distinguished according to their $\mathrm{THz}$ fingerprints. With $<5-\mathrm{mW}$ optical power, the demonstrated system allows us to specify different illicit drug powders with weights of nanograms.

Fig. 1(a) illustrates the schematic diagram of the proposed THz-biosensing chip with a size of $1 \mathrm{~mm} \times 1 \mathrm{~mm}$. The top part is the glass-substrated polyethylene (PE) sample cell with hot-embossed [8] 20- $\mu \mathrm{m}$-deep channels where sample powders were contained. The weights of the powders inside the detection channel were on the order of $300 \mathrm{ng}$, estimated by an area ratio method. A broadband $\mathrm{THz}$ photonic transmitter was inversely integrated in the bottom of the $150-\mu \mathrm{m}$-thick glass substrate. The layout of the photonic transmitter is shown in Fig. 1(b) [9]. The 5- $\mu \mathrm{m}$-thick photonic transmitter is composed of an edgecoupled metal-semiconductor-metal traveling-wave photodetector (MSM TWPD), [10] coplanar-waveguides (CPWs), and a slot antenna [11], [12]. With an edge-coupled structure, illumination light with a $\mathrm{THz}$ beating frequency can be completely absorbed in the detector while high electrical bandwidth is preserved through a traveling-wave structure [13]. The light-driven 


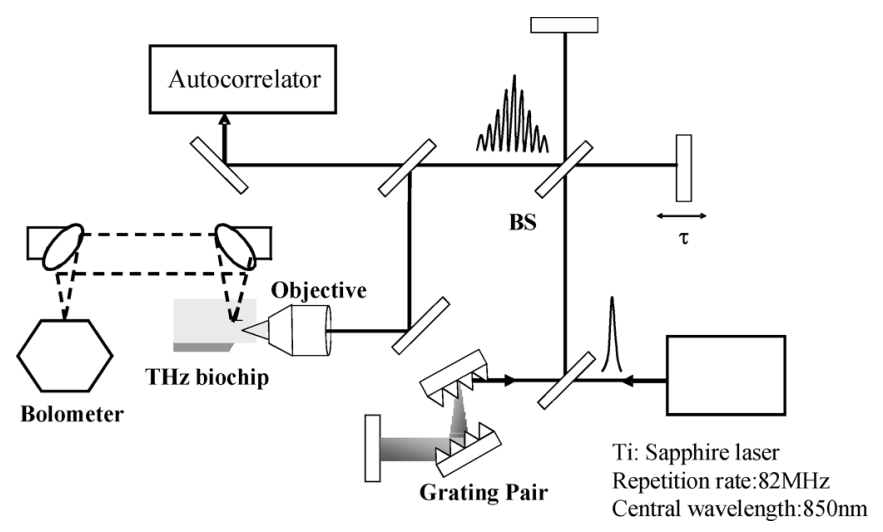

Fig. 2. Schematic diagram of the THz spectral measurement system. BS: beam splitter. Solid line: excitation beam. Dashed line: radiated quasi-CW THz beam.

photocurrent output, with a $\mathrm{THz}$ frequency corresponding to the optical beating frequency, propagated through the CPW and fed into the slot dipole antenna for $\mathrm{THz}$ radiation. Through simulation (HFSS v10.0, Ansoft Company), the transverse electromagnetic (TEM) lines along the MSM TWPD and the CPW are confirmed to be well confined inside the glass, and have no effect on the tested powders. A wide tuning range from $<100 \mathrm{GHz}$ to $1.15 \mathrm{THz}$ was demonstrated from a single photonic transmitter [9], while this glass-substrated device exhibited an exceptional high light-to-THz external power conversion efficiency of $0.33 \%$ [9]. Hence, under milliwatt average power optical excitation, the output average $\mathrm{THz}$ power after the highly absorptive glass was on the order of microwatts. By placing the microsample cell parallelly aligned right on top of the radiating slot antenna within a near-field distance of $150 \mu \mathrm{m}$, which is shorter than the $\mathrm{THz}$ wavelength, we are thus able to increase the THz photon flux at the samples as well as the percentage of the $\mathrm{THz}$ radiation experiencing the testing powders, achieving localized sensing with high sensitivity.

Fig. 2 shows the layout of the $\mathrm{THz}$ spectral measurement system, where we used a mode-locked Ti : sapphire laser and an optical coherent control system [7] for radiating frequency control. The grating pair stretched the 100 -fs pulses at $800 \mathrm{~nm}$ with an $82-\mathrm{MHz}$ repetition rate into linearly chirped pulses with a desired pulsewidth. By controlling the time delay of the two arms in a Michelson interferometer, we could control optical beating frequency [7], which is linearly proportional to the arm length difference. The beating light with an average power $<5 \mathrm{~mW}$ was then focused into the MSM TWPD. The quasi-continuous-wave $(\mathrm{CW}) \mathrm{THz}$ emission from the antenna, of which the radiation frequency and linewidth were controlled by the optical coherent control system [14], transmitting through the microsample cell in a $\mathrm{THz}$ near-field distance from the antenna, were collected by a pair of parabolic mirrors and a Si-bolometer for power detection. By inserting a chopper in the optical path and connecting the bolometer output to a lock-in, the background noise was reduced. We acquired the $\mathrm{THz}$ emission spectra $(\mathrm{THz}$ transmission intensity versus emission frequency) from the microchip with and without the tested powders by varying the optical beating frequency through the coherent control system. An HFSS simulation considering only the refractive index effect, neglecting the absorption constant of

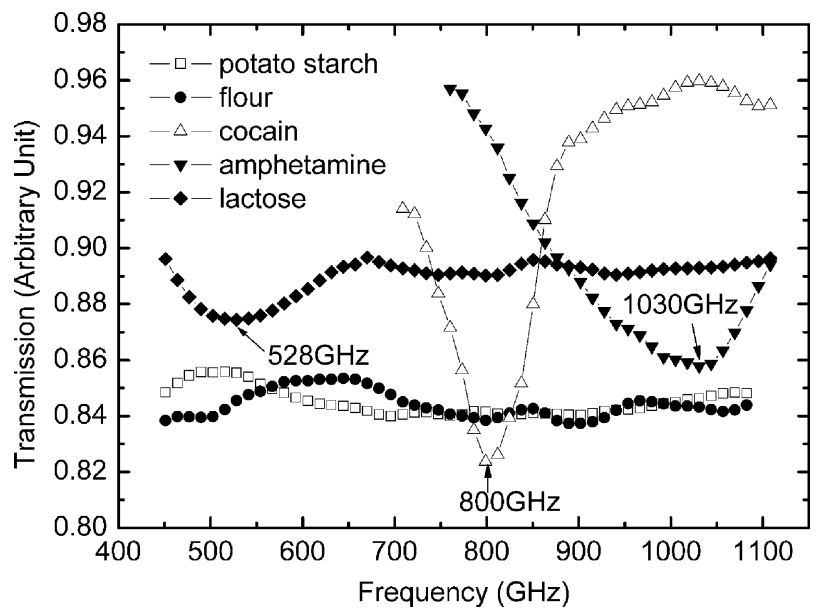

Fig. 3. THz microabsorption spectra of five different white powders tested, acquired by the $\mathrm{THz}$ microchip system. Two of them are illicit drugs including cocaine and amphetamine.

the tested powders, shows no significant (less than the system noise level) radiation power variation by adding different tested powders. Dividing the collected $\mathrm{THz}$ power with and without the samples at different frequencies, we thus acquired the $\mathrm{THz}$ absorption microspectrum of a specific drug powder, which directly reflects the vibrational nature of the tested molecules. Compared with the time-domain $\mathrm{THz}$ system, the spectral domain system is with a narrower emission bandwidth and thus with a higher spectral power density. The frequency domain system also possesses the unique characteristic that the acquired spectral range and resolution can be controlled, thus shortening the data acquisition time. With an optical pulsewidth of $10 \mathrm{ps}$ and an excitation power of $4.8 \mathrm{~mW}$, the signal-to-noise ratio (SNR) of the complete $\mathrm{THz}$ system was about 300 at $1 \mathrm{THz}$ with a lock-in integration time of $100 \mathrm{~ms}$. The SNR is better than 300 for the sub-THz regime. The obtained SNR can be further enhanced with higher optical excitation power, improved light-THz conversion efficiency, a longer integration time, or by shortening the distance between the microchip and the bolometer.

Fig. 3 shows the acquired $\mathrm{THz}$ absorption microspectra of five different white powders. With a finite scanning range and a reasonable spectral resolution $(50 \mathrm{GHz}$ in this specific case for faster scanning speed), the acquisition time of all traces can be within 1-5 s in our current system. With $300 \mathrm{ng}$ of powders, the characteristic absorption peaks of cocaine (cocainae hydrochloridum, National Bureau of Controlled Drugs, Taiwan, R.O.C.) and amphetamine (methamphetamine, alpha-dimethylphenethylamine, National Bureau of Controlled Drugs, Taiwan, R.O.C.) at $\sim 800$ [15] and $\sim 1030 \mathrm{GHz}$ [15] can both be clearly identified, in sharp contrast to the potato starch, flour, and lactose powders. The latter has a $530-\mathrm{GHz}$ [16] absorption peak. The low average transmission of potato starch and flour is due to the hexagonal molecular crystalline pattern which traps water molecules [17]. With the measured transmission variation, our system showed a 10-ng detection limit for cocaine and amphetamine powders under $4.8-\mathrm{mW}$ excitation and with 100-ms integration time. The measurements were also repeated for stability test. High system stability can 
be found. Taking amphetamine spectra as an example, the maximum frequency shift of the measured absorption peak was found to be one scan step (6 GHz for the trace in Fig. 3), which is much smaller than the frequency resolution of our system. This result indicates that our $\mathrm{THz}$ microchip system can provide the capability to identity different illicit drug powders with a minute amount (on the order of or less than $10 \mathrm{ng}$ ), which is with a sensitivity comparable to the current forensic toxicology identification techniques, such as fluorescence polarization immunoassay [18] and gas chromatography-mass spectrometry [19] with a detection limit of $100-500 \mathrm{ng} / \mathrm{ml}$. With much improved sensitivity in the future, optoelectronic-integrated $\mathrm{THz}$ microchips should also enable water-based molecular sensing due to featureless and tolerable water absorption [20] through a 20- to 50- $\mu \mathrm{m}$-thick microfluidic channel. With the detected molecules preserved in their original states, optoelectronic-integrated $\mathrm{THz}$ biochip should be able to provide the desired capability for future molecular dynamics studies.

In conclusion, we demonstrated the feasibility to integrate an optoelectronic-based micro- $\mathrm{THz}$ source with a glass-substrated microchip, with the sample in the near-field distance of the $\mathrm{THz}$ radiation antenna for localized biosensing. The capability to identity different illicit drug powders based on their spectral characteristics was successfully demonstrated with a $<10$ nanogram sensitivity. This $\mathrm{THz}$ microchip system has the advantages of label-free detection (need no probing molecules), high selectivity (based on spectral characteristics of molecules), no sample preparation, repeatable usage (just to replace the PE film), and ease of parallel integration with other biochip functionality modules, which are desirable for future lab-on-a-chip applications. In addition, by placing the detected molecules within the near-field distance of the micro-THz source, localized detection can be achieved, having the potential in multiplexing for future array applications. Our demonstration shows the possibility to integrate optoelectronic-based photonic transmitters with the current biochip technology for various biosensing applications, including DNA sequencing, explosive and virus detections. Due to the unique capability to detect the biomolecules in their natural states, the $\mathrm{THz}$ biochip should also enable future studies of molecular conformational dynamics.

\section{ACKNOWLEDGMENT}

The authors would like to thank Prof. M.-C. Tsai and Prof. C.-L. Pang for technical support.

\section{REFERENCES}

[1] H. Ozaki and L. W. McLaughlin, "The estimation of distances between specific backbone-labeled sites in DNA using fluorescence resonance energy transfer," Nucleic Acids Res., vol. 20, pp. 5205-5214, 1992.
[2] M. Nagel, F. Richter, P. Haring-Bolívar, and H. Kurz, "A functionalized THz sensor for marker-free DNA analysis," Phys. Med. Biol., vol. 48, pp. 3625-3636, 2003.

[3] A. Menikh, R. MacColl, C. A. Mannella, and X. C. Zhang, "Terahertz biosensing technology: Frontiers and progress," Chemphyschem, vol. 3, pp. 655-658, 2002.

[4] F. Stewing, T. Kleine-Ostmann, and M. Koch, "A new class of improved efficiency $\mathrm{THz}$ filters for onchip detection of biomaterials," $\mathrm{Mi}$ crow. Opt. Technol. Lett., vol. 41, pp. 79-82, 2004.

[5] N. Flanders, R. A. Cheville, D. Grischkowsky, and N. F. Scherer, "Pulsed terahertz transmission spectroscopy of liquid CHCl3, CCL4, and their mixtures," J. Phys. Chem., vol. 100, pp. 11824-11835, 1996.

[6] T. Globus, D. L. Woolard, T. Khromova, T. W. Crowe, M. Bykhovskaia, B. L. Gelmont, J. Hesler, and A. C. Samuels, "THz-spectroscopy of biological molecules,” J. Biol. Phys., vol. 29, pp. 89-100, 2003.

[7] A. S. Weling and D. H. Auston, "Novel sources and detectors for coherent tunable narrowband terahertz radiation in free space," J. Opt. Soc. Amer. B, vol. 13, pp. 2783-2791, 1996.

[8] G.-B. Lee, S.-H. Chen, G.-R. Huang, W. C. Sung, and Y.-H. Lin, "Microfabricated plastic chips by hot embossing methods and their applications for DNA separation and detection,"Sens. Actuator B, Chem., vol. 75, pp. 142-148, 2001.

[9] T.-F. Kao, H.-H. Chang, L.-J. Chen, J.-Y. Lu, A.-S. Liu, Y.-C. Yu, R.-B. Wu, W.-S. Liu, J.-I. Chyi, and C.-K. Sun, "Frequency tunability of terahertz photonic transmitter," Appl. Phys. Lett., vol. 88, p. 093501, 2006.

[10] J.-W. Shi, K.-G. Gan, Y.-J. Yang, Y.-H. Chen, C.-K. Sun, Y.-J. Yang, and J. E. Bowers, "Metal-semiconductor-metal traveling-wave photodetectors," IEEE Photon. Technol. Lett., vol. 13, no. 6, pp. 623-625, Jun. 2001.

[11] J.-W. Shi, S.-W. Chu, M.-C. Tien, C.-K. Sun, Y.-J. Chiu, and J. E. Bowers, "Edge-coupled membrane terahertz photonic transmitters based on metal-semiconductor-metal traveling photodetectors," Appl. Phys. Lett., vol. 81, pp. 5108-5110, 2002.

[12] M.-C. Tien, H.-H. Chang, J.-Y. Lu, L.-J. Chen, C.-Y. Chen, R.-B. Wu, W.-S. Liu, J.-I. Chyi, and C.-K. Sun, "Device saturation behavior of submillimeter-wave membrane photonic transmitters," IEEE Photon. Technol. Lett., vol. 16, no. 3, pp. 873-875, Mar. 2004.

[13] J.-W. Shi, K.-G. Gan, Y.-H. Chen, C.-K. Sun, Y.-J. Chiu, and J. E. Bowers, "Ultrahigh-power-bandwidth product and nonlinear photoconductance performances of low-temperature-grown GaAs-based metal-semiconductor-metal traveling-wave photodetectors," IEEE Photon. Technol. Lett., vol. 14, no. 11, pp. 1587-1589, Nov. 2002.

[14] L.-J. Chen, T.-F. Kao, J.-Y. Lu, and C.-K. Sun, "A simple terahertz spectrometer based on a low-reflectivity Fabry-Pérot interferometer using Fourier transform spectroscopy," Opt. Express, vol. 14, pp. 3840-3846, 2006.

[15] K. Kawase, Y. Ogawa, H. Minamide, and H. Ito, "Terahertz parametric sources and imaging applications," Semicond. Sci. Technol., vol. 20, pp. S258-S265, 2005.

[16] B. Fischer, M. Hoffmann, H. Helm, G. Modjesch, and P. U. Jepsen, "Chemical recognition in terahertz time-domain spectroscopy and imaging," Semicond. Sci. Technol., vol. 20, pp. S246-S253, 2005.

[17] T. L. J. Chan, J. E. Bjarnason, A. W. M. Lee, M. A. Celis, and E. R. Brown, "Attenuation contrast between biomolecular and inorganic materials at terahertz frequencies," Appl. Phys. Lett., vol. 85, pp. 2523-2525, 2004.

[18] T. Kraemer, G. A. Theis, A. A. Weber, and H. H. Maurer, "Studies on the metabolism and toxicological detection of the amphetamine-like anorectic fenproporex in human urine by gas chromatography-mass spectrometry and fluorescence polarization immunoassay," J. Chromatogr. B: Biomed. Sci. Appl., vol. 738, pp. 107-18, 2000.

[19] R. H. Williams, J. A. Maggiore, S. M. Shah, T. B. Erickson, and A. Negrusz, "Cocaine and its major metabolites in plasma and urine samples from patients in an urban emergency medicine setting," J. Anal. Toxicol., vol. 24, pp. 478-481, 2000.

[20] L. Thrane, R. H. Jacobsen, R. H. U. Jepsen, and S. R. Keiding, "THz reflection spectroscopy of liquid water," Chem. Phys. Lett., vol. 240, pp. 330-333, 1995. 